\title{
WILEY-VCH
}

\section{D Printed Robotic Assembly Enabled Reconfigurable Display with Higher Resolution}

\author{
Nadeem Qaiser, Sherjeel Munsif Khan, Kelvin Chow, Marlon Diaz Cordero, \\ Irmandy Wicaksono and Muhammad Mustafa Hussain ${ }^{1 *}$ \\ ${ }^{1}$ mmh labs, Electrical Engineering, Computer Electrical and Mathematical Science and \\ Engineering Division, King Abdullah University of Science and Technology (KAUST) \\ Thuwal, 23955, Saudi Arabia, Tel.: +966-544-700-072
}

*Corresponding author; E-mail: muhammadmustafa.hussain@kaust.edu.sa

Keywords: stretchable electronics, pixel resolution, reconfigurable display, FEM analysis, $3 D$ printing

\begin{abstract}
The stretchable display might play a crucial role to transform the potential application including wearable electronics, flexible display for smart TV/devices, health monitoring wristbands and illumination systems. To date, the most commonly used stretchable display include the installation of LEDs onto the compliant substrate. However, they have critical limitations such as an increase in resistance and degradation of pixel resolution due to growing gaps between LEDs. Here, we demonstrate a reversible stretchable platform, which preserves not only the resistance of LEDs but also the pixel resolution of the display. Our stretchable platform utilizes the concept of the multilevel arrangement of LEDs, which are mechanically guided by joint pins/links to move out-ofplane and fill the evolved gap. To corroborate the concept of the reconfigurable display, we manufacture the reconfigurable platform by using a 3D printer. Our design might be a key enabling technology to next-generation expandable display and illuminations systems.
\end{abstract}




\section{WILEY-VCH}

\section{Introduction}

Stretchable electronics has sustained its worth in various applications such as sensory skins for robotics, wearable communication devices, optoelectronics, bio-medicines/bio-integrated devices, bendable and flexible display. ${ }^{[1-5]}$ The flexible electronic display and image sensors have recently gained extensive attention, ${ }^{[5-12]}$ due to various benefits, which include the improved functionalities for daily-life work, better durability, lighter weight, curved design feature and thinner dimensions. Moreover, various technological advancements presently have paved a way to attain novel designs and improved engineering prototypes, which result in stateof-the-art devices such as wearable health monitoring bands, electronic paper, and displays-onwrists. Currently, researchers have demonstrated extensive work in the area of CMOS based stretchable sensors/actuators and wearable sensors, ${ }^{[13-17]}$ however, there is still a lack of understanding in the area of the stretchable and size-variable display. As a futuristic application, we can envision a next-generation display or solid-state lighting system, which could change its size or typically make reconfigurable itself. For instance, by utilizing highly stretchable or expandable display, a small screen-sized mobile phone may be rehabilitated into large screensized tab or laptop. Likewise, we can attain a stretchable and fashionable electronic clothing with built-in electronic functionalities and biocompatible light sources by simply stretching the display. The potential applications of these devices consist of the stretchable in vivo medical devices/robotic systems, multi-functional expandable mobile phones, smart TV, and illumination systems. A conceptual outline and associated problem with existing Ecoflexsubstrate based stretchable device are shown in Fig. 1.

As of today, most commonly used strategies to attain the stretchable platforms for display include the combination of LEDs with soft and rubbery materials i.e. placing rigid LEDs along 


\section{WILEY-VCH}

with elastic interconnects onto a soft substrate or using compliant LEDs that are intrinsically stretchable. ${ }^{[18-20]}$ For instance, M.S. White et al. demonstrated a display-compatible ultrathin (2 $\mu \mathrm{m})$ red and orange polymer light-emitting-diodes (LEDs), which showed the enhanced mechanical stability. ${ }^{[21]}$ Although the abovementioned systems have demonstrated the promising results in terms of stretchability and efficiency, these devices have critical limitations. Firstly, during stretching the electrical resistance of stretchable electrodes increases under tensile stresses. Previous studies have proposed various techniques to resolve the stated issue i.e. perforated 3D net-shaped nanostructures in the PDMS, ${ }^{[22]}$ inkjet-printed stretchable silver electrodes, ${ }^{[23]}$ and using various interconnects with/without embedded PDMS. ${ }^{[24,25]}$ Nevertheless, the outcome and efficiency of these devices are still far below than the essential level. Secondly and most importantly, devices that comprise the stretchable display, experience the degraded or lower pixel resolution during their operation. The reason for this deficiency could be regarded to the growing gaps between simultaneous LEDs or lower pixel density when expandable displays stretch out. Therefore, to get the highly efficient reconfigurable display a more realistic and novel platform is needed.

Here, we propose, a reconfigurable and size-variable platform, which is capable to provide the highly efficient display without affecting the pixel resolution/density and electrical resistance of LEDs. This reconfigurable prototype utilizes the notion of multi-level LEDs i.e. placing the LEDs arrays on top of another in the stack. During stretching, these stacked arrays of LEDs move upward or out-of-plane to fill the evolved gaps, which is reversible and preserves the original resolution simultaneously. We performed numerical modeling to demonstrate that the induced stresses and strains were readily insignificant and thus creating the system mechanically highly reliable. We have tested our reconfigurable platform by using the 3D 


\section{WILEY-VCH}

printer. We installed the LEDs on the platform and showed no loss in pixel resolution, which proved the performance of our design. The proposed platform would lay the groundwork for promising future with revolutionary display and illumination systems without losing their performance and resolution/image quality.

\section{Results and Discussions}

Figure 1 shows the conceptual outline for a representative display that is capable to expand or stretch without degradation of image quality or resolution. For instance, the mobile phone has originally a display of size $\mathrm{x}$, which may possibly transform into an IPad or Tab by extending its display size up to 2x as shown in Fig. 1a and 1b. Generally, the image quality and performance of a display is characterized by the number of LEDs it contains and no-gap between these LEDs, as shown in Fig. 1c. In other words, if there is any LED missing, the color of the display will be black at that position and thus the quality will decrease. Now, let us postulate that we install a set of LEDs onto a flexible substrate (i.e. complaint Ecoflex) and stretch out the feature. As a result, LEDs are anticipated to move/change their positions and gap between any two consecutive LEDs will grow, as shown in Fig. 1d. These evolved gaps would affect the resolution or quality of a typical display.

In order to achieve the stretchable displays, various strategies were previously explored, however, these designs have a few critical limitations i.e. increase in the resistance of LEDs and the degradation of the resultant resolution during stretching. ${ }^{[18,19]}$ On the subject of growing gaps, the major reason could be attributed to the expansion of device in only one direction i.e. in-plane movements, through which a realistic display having gap-free LEDs is nearly impossible. Here, we devise a novel design that contains the multi-level stacks or layers of LEDs. Figure 2a and 2b 


\section{WILEY-VCH}

reveal the front and 3D view of the design, which is capable to act as an expandable and reconfigurable display by moving the layers out-of-plane or upward. As the LEDs might be positioned onto the surface of square islands, arrangement of square islands are supposed to represent the corresponding LEDs. The width and height of these islands are expressed as b and t, while the total height of the assembled platform is expressed as $h_{p}$, respectively. The thickness of island and height of the platform is taken as $\mathrm{b} / 10$ and $2 \mathrm{~b}$, respectively. In addition to islands located at top-level, feature contains three more levels (from $1^{\text {st }}$ to $3^{\text {rd }}$ level) connected mechanically thorough links and pins.

The certain arrangement of islands, links, and pins guide the islands of underneath level to move out-of-plane and fill the gap, as shown in Fig. 2c to 2e. For instance, platform initially has 4 islands (2x2 array) representing the original size and during stretching when these islands start to displace along the $\mathrm{x}$-axis, a gap will be evolved due to change in their positions. However, due to the specific design, the two islands of the $1^{\text {st }}$ level will move upward to fit into the evolved gap, thus forming a $2 \times 3$ array (6 islands), as shown in Fig. 2d. Likewise, the stretching along y-axis would cause the out-of-plane movement of islands of $2^{\text {nd }}$ and $3^{\text {rd }}$ levels. As a result, the $3 \times 3$ gapfree array will be accomplished from a primary 2x2 array, as shown in Fig. 2e. The major benefit of our platform is that might provide no or negligible change in resolution. Moreover, as the LEDs will not experience any strain/stress LED’s resistance will be unaffected.

As a next step, we validate the functioning of the stretchable platform by using the 3D printed platforms. We also checked the scalability of the platform by importing the design with a diverse sizes i.e. different height of platform hp and width of square-island b. Figure 3a shows the set of printed platforms with different values of $b$ (inset shows a front and cross-sectional view of typical display device). The range of $\mathrm{b}$ was chosen from $\sim 40 \mathrm{~mm}$ to $\sim 5 \mathrm{~mm}$. Figure $3 \mathrm{~b}$ reveals the 


\section{WILEY-VCH}

linear relationship between $h_{p}$ and $b$, as expected, thus shows the promising scalability of the platform. The smallest printed platform has $h_{p}$ of $\sim 10 \mathrm{~mm}$, which is comparable to the display size of existing display devices such as laptop, TV and even mobile phones. Figure 3c demonstrates the functioning of the reconfigurable platform. The platform stretch to enlarge its size and fold to revert to the original size that makes it completely reversible.

It should be noted that due to the lack of sophisticated tools, handling of the smaller platforms ( i.e. when b is less than $15 \mathrm{~mm}$ ) remains challenging. Therefore, in order to simplify the handling of the stretching/folding process, we selected the size of $\sim \mathrm{b}=15 \mathrm{~mm}$. However, with the state-of-the-art technology in the areas of MEMS, particularly in micro-machines, we may anticipate that the platform with micron-sized range would be promising and pave a way to transform the current display into size-variable and reversible displays. Moreover, various application may be covered by connecting these platforms to acquire the extended version e.g. applicable for increased no. of pixels. These extended-links might be positioned purposefully in such a way that LEDs do not interfere or overlap with each other. For instance, one possible way is integrating these unit-cells in-series and then out-of-plane motions of LEDs on each of the $2^{\text {nd }}$ and $3^{\text {rd }}$ levels can be controlled by using a long guiding-rail on top and bottom edges of the display. As a result, all of the LEDs will move upward simultaneously and definitely will not overlap with another. Figure 3c demonstrates the stretching and folding process of the reconfigurable platform. From the initial size of the $2 x 2$ array, the platform is stretched along the $y$-axis to transform into the $2 \times 3$ array. Likewise, the $2 \times 3$ array transforms into a larger size of the $3 \times 3$ array by stretching along the x-axis. In contrast, size of platform reverts to the original size by folding process i.e. from the $3 x 3$ array to $2 x 3$ along the $x$-axis, and then to $2 x 2$ array along the $y$-axis, respectively. The stretching and folding of the platform prove the reversibility of it. Details of stretching and 


\section{WILEY-VCH}

folding can be seen in SMovie1 of supplementary information. It is clear from the Fig. 3c and SMovie1 that the islands move out-of-the-plane to fill the evolved gaps and thus are expected to sustain the original resolution or image quality.

An important criterion that needs to be considered is the estimation of strength or resistance of reconfigurable platform against the crack-based failure during the stretching process. The induced stress depends on the relative motion between each of the component, geometry and corresponding feature size i.e. islands, links, joint pins. Therefore, to map the induced stresses/strains, a numerical analysis by using the Multibody Dynamics module of COMSOL ${ }^{\mathrm{TM}}$ was performed. To replicate the stretching process described in Fig. 3c, we stretched the assembled platform along the $\mathrm{x}$-axis and $\mathrm{y}$-axis, respectively. Figure $4 \mathrm{a}$ and $4 \mathrm{~b}$ exhibit the displacement distributions while transforming the 2x2 arrays (4 islands) into a 2x3 array (6 islands) and then into a 3x3 array (9 islands), respectively. The arrow (green colored) represent the direction of motions of islands and links (For stretching and array transformation process see the supplementary video SMovie2 and SMovie3).

The mechanical response of structure or failure criterion under tensile forces might be described by the magnitude of von Mises stress and strains. For instance, when the maximum von Mises stress in the structure is lower than the yield point of material ( 1GPa for $\mathrm{Si}$ ), it may avoid the growth or propagation of cracks. It is logical to say that the platform experiences the maximum von Mises stress at onset of fully stretched state. Fig. 4c reveals the stress contours at fully stretched state. It is clear from the Fig. that the islands (likewise LEDs) have negligible stress, while pins and links experience the maximum von Mises stress. However, the magnitude of induced maximum von Mises stress is found to be less than $5 \mathrm{MPa}$ that is very low as compared to the yield strength of the material. 


\section{WILEY-VCH}

Figure 4d demonstrates the history of von Mises stress at one node as a function of prescribed displacement. The inset of Fig. 4d shows the position of that particular node. It is clear from the Fig. 4d that the structure experiences the maximum von Mises stress at the end of stretching process since all of the solid components of the platform have unconstrained motions. The strain distributions and in-depth stresses in links and pins are shown in supplementary Fig. S1 of supplementary information, which show that platform has a small magnitude of strains and stresses. The results validate that our reconfigurable platform has higher mechanical reliability as compared to previous schemes, wherein only in-plane motions occur during stretching. ${ }^{[8,22,24]}$

Next, in order to test the performance of our reconfigurable platform, we installed the LEDs onto our platform. To represent the initial size of the display, we installed the green colored LEDs on the top level and red colored LEDs on lower levels would represent the upward movement of lower-level LEDs. As the commercially available LEDs were a bit smaller in size as compared to chosen size of island i.e. $b=15 \mathrm{~mm}$, we placed the thin polymeric lens on top of LEDs to provide the lucid presentation of proposed platform. Figure 5 shows the performance of our expandable and reconfigurable platform. The reason for blurry image can be attributed to the presence of polymeric lens on top of the LEDs. The details of the reversible process of stretching and folding can be seen in SMovie4 of supplementary information. Fig. 5a-5c reveals that the size of display increases from the $2 \times 2$ array to $2 \times 3$ array, and then to the $3 \times 3$ array, by stretching along $y$-axis and $\mathrm{x}$-axis, respectively. Likewise, the reverting back to the original size is achieved by the folding of the platform, as shown in Fig. 5d-5f.

It was quite challenging to handle the LEDs testing manually, especially by using tools with limited functionalities. When we manually stretch the platform integrated with LEDs, we apply the tensile forces in both axis/direction in certain order, which might cause a backlash during 


\section{WILEY-VCH}

stretching/folding process. As a result, it generated an effect of tilting observed in figure 5. Nevertheless, it does keep its position as guided by links, which is evidenced by the FEM and experimental work shown in $\sim$ SMovie1 to $\sim$ SMovie4. Our results establish the fact that the proposed platform maintains the original resolution and higher mechanical reliability, which will pave a way to futuristic expandable, size-variable display and illumination systems.

\section{Conclusions and Summary}

In conclusion, we have devised a novel platform for a stretchable and reconfigurable display, which could maintain the original pixel resolution of the display. Moreover, our design is anticipated to experience no change in the resistance of LEDs. The stretchable platform consists of LEDs positioned at multi-levels in the stack, which are guided by mechanical links and pins. Generally, during stretching the LEDs move apart and evolve a gap, thus degrade the pixel resolution. However, our design has LEDs on multi-level and the particular arrangement of components of our design utilized the out-of-plane or upward movements of LEDs to fill the evolved gap during stretching. To check the reliability of our platform, we used numerical simulations to calculate the von Mises stress and strain. Results showed that the maximum von Mises stress was found to be very insignificant i.e. 5 MPa in links and pins region, which ensured the resistance to potential failure based on cracks growth or propagation.

To corroborate our design, we used 3D printing to manufacture the replica of the stretchable and reconfigurable display. The printed design was able to stretch its size from $2 x 2$ array, to $2 x 3$ array and then $3 \mathrm{x} 3$ array, when stretched along y-axis and $\mathrm{x}$-axis, respectively. Similarly, it reverted to original state from fully stretched state, thus making it fully reversible. To show the proof of concept, we installed the LEDs onto the platform, which was effectively able to stretch 


\section{WILEY-VCH}

and fold without losing the resolution. The performance of our reconfigurable platform shows that it might be very useful for next-generation display and illumination systems, especially diverse range of applications can be addressed by connecting these platforms in-series. It is noteworthy to mention that the proposed design has yet not reached to the level of commercialization since it might has few limitations such as high device volume due to movable components in layers and respective links. Additionally, to acquire the realistic display applicable for smart and wearable devices, limitation of platform height should be resolved in a well manner.

\section{Materials and Methods}

\subsection{Design and Simulation}

We used SOLIDWORKS ${ }^{\mathrm{TM}}$ to design the individual 3D components of reconfigurable platform including the islands, pins and links and then imported these 3D solid parts in assembly module to acquire the fully assembled platform. The thickness of square-island and height of platform were taken as $b / 10$ and $2 b$, respectively, whereas $b$ is the width of square-island. It should be noted that in order to demonstrate the scalability of the platform, we chose different values of $\mathrm{b}$, ranging from $\sim 40 \mathrm{~mm}$ to $5 \mathrm{~mm}$. We used a commercially available numerical tool $\mathrm{COMSOL}^{\mathrm{TM}}$ to calculate the stress/strain behavior during stretching. As the final assembled design is composed of different 3D components, which are connected through mechanical pins and have relative motions, the numerical solution needs to cover the complex dynamics. COMSOL $^{\mathrm{TM}}$ has a special module, which solves this sort of complex problems called Multibody Dynamics. We reproduced the reconfigurable platform in COMSOL and defined the hinge and prismatic joints wherever needed in the design. We used the isotropic Si as a linear elastic material. The material properties of Si were taken from previous study. ${ }^{[16]}$ 


\section{WILEY-VCH}

In order to get the stress response under stretching, we prescribed a displacement of b i.e. equivalent to a size of island. We imposed the displacement on one side of platform and fixed the opposite side. To mimic the real device operation, we executed the FEM simulations in two steps i.e. first prescribed the displacement along $\mathrm{x}$-axis and then same amount was prescribed along y-axis, respectively. To make sure that the solution is converged, a sufficiently fine mesh with triangular elements was used. A stationary solver with sweep function was employed.

\subsection{Experiments}

We used the ProJet MJP 2500 Plus 3D printer by 3dsystems to print the 3D design of reconfigurable platform. The material provided by 3dsystems called WisiJet M2R-WT was used. We imported the 3D assembled designs to the printer with diverse range of $b$ from SOLIDWORKS ${ }^{\mathrm{TM}}$. After cleaning, these printed specimens were subject to stretching. In order to demonstrate the concept of stretchable display without the loss of display resolution. We attached Xlamp MC-E LED multichip LEDs that give high lumen output in a small package. They are multicolour LEDs so one package can be used to give different colours. We used multicolour LEDs to assign different colours to each levels so new pixels can be seen clearly, when the LEDs from lower level move out-of-the-plane during stretching. Top-level pixels were set to green colour while others pixels were set to red colour. Agilent E3631A DC Power Supply powers the LEDs at $3 \mathrm{~V}$ with a controller current of $20 \mathrm{~mA}$.

\section{Supplementary Material}

See supplementary material for reversible stretching and folding process of 3D printed reconfigurable platform, 3D strain contours in platform and stress distribution in pins/links, array 


\section{WILEY-VCH}

transformation process with stretching and folding process by FEM, and fully functional platform with LEDs.

\section{Acknowledgment}

This publication is based upon work supported by the King Abdullah University of Science and Technology (KAUST) Office of Sponsored Research (OSR) under KAUSTKFUPM Special Initiative Award No. OSR-2016-KKI-2880.

\section{References}

[1] S. Choi, H. Lee, R. Ghaffari, T. Hyeon, D.-H. Kim, Adv. Mater. 2016, 28, 4203.

[2] J. Viventi, D.-H. Kim, J. D. Moss, Y.-S. Kim, J. A. Blanco, N. Annetta, A. Hicks, J. Xiao, Y. Huang, D. J. Callans, J. A. Rogers, B. Litt, Sci. Transl. Med. 2010, 2.

[3] M. Ramuz, B. C.-K. Tee, J. B.-H. Tok, Z. Bao, Adv. Mater. 2012, 24, 3223.

[4] B. Tian, J. Liu, T. Dvir, L. Jin, J. H. Tsui, Q. Qing, Z. Suo, R. Langer, D. S. Kohane, C. M. Lieber, Nat. Mater. 2012, 11, 986.

[5] J.-S. Park, T.-W. Kim, D. Stryakhilev, J.-S. Lee, S.-G. An, Y.-S. Pyo, D.-B. Lee, Y. G. Mo, D.-U. Jin, H. K. Chung, Appl. Phys. Lett. 2009, 95, 013503.

[6] R.-H. Kim, D.-H. Kim, J. Xiao, B. H. Kim, S.-I. Park, B. Panilaitis, R. Ghaffari, J. Yao, M. Li, Z. Liu, V. Malyarchuk, D. G. Kim, A.-P. Le, R. G. Nuzzo, D. L. Kaplan, F. G. Omenetto, Y. Huang, Z. Kang, J. A. Rogers, Nat. Mater. 2010, 9, 929. 


\section{WILEY-VCH}

[7] M. K. Choi, J. Yang, T. Hyeon, D.-H. Kim, npj Flex. Electron. 2018, 2, 10.

[8] T. Sekitani, H. Nakajima, H. Maeda, T. Fukushima, T. Aida, K. Hata, T. Someya, Nat. Mater. 2009, 8, 494.

[9] T.-H. Han, Y. Lee, M.-R. Choi, S.-H. Woo, S.-H. Bae, B. H. Hong, J.-H. Ahn, T.-W. Lee, Nat. Photonics 2012, 6, 105.

[10] J. H. Koo, D. C. Kim, H. J. Shim, T.-H. Kim, D.-H. Kim, Adv. Funct. Mater. 2018, 28, 1801834.

[11] M. K. Choi, J. Yang, D. C. Kim, Z. Dai, J. Kim, H. Seung, V. S. Kale, S. J. Sung, C. R. Park, N. Lu, T. Hyeon, D.-H. Kim, Adv. Mater. 2018, 30, 1703279.

[12] C. Choi, M. K. Choi, S. Liu, M. S. Kim, O. K. Park, C. Im, J. Kim, X. Qin, G. J. Lee, K. W. Cho, M. Kim, E. Joh, J. Lee, D. Son, S.-H. Kwon, N. L. Jeon, Y. M. Song, N. Lu, D.H. Kim, Nat. Commun. 2017, 8, 1664.

[13] J. M. Nassar, J. P. Rojas, A. M. Hussain, M. M. Hussain, Extrem. Mech. Lett. 2016, 9, 245.

[14] J. P. Rojas, G. A. Torres Sevilla, M. T. Ghoneim, S. Bin Inayat, S. M. Ahmed, A. M. Hussain, M. M. Hussain, ACS Nano 2014, 8, 1468.

[15] A. M. Hussain, E. B. Lizardo, G. A. Torres Sevilla, J. M. Nassar, M. M. Hussain, Adv. Healthc. Mater. 2015, 4, 665.

[16] N. Qaiser, S. M. Khan, M. Nour, M. U. Rehman, J. P. Rojas, M. M. Hussain, Appl. Phys. Lett. 2017, 111, 214102.

[17] J.-K. Song, D. Son, J. Kim, Y. J. Yoo, G. J. Lee, L. Wang, M. K. Choi, J. Yang, M. Lee, 


\section{WILEY-VCH}

K. Do, J. H. Koo, N. Lu, J. H. Kim, T. Hyeon, Y. M. Song, D.-H. Kim, Adv. Funct. Mater. 2017, 27, 1605286.

[18] H. L. Filiatrault, G. C. Porteous, R. S. Carmichael, G. J. E. Davidson, T. B. Carmichael, Adv. Mater. 2012, 24, 2673.

[19] T. Someya, Nat. Mater. 2010, 9, 879.

[20] J. Liang, L. Li, X. Niu, Z. Yu, Q. Pei, Nat. Photonics 2013, 7, 817.

[21] M. S. White, M. Kaltenbrunner, E. D. Głowacki, K. Gutnichenko, G. Kettlgruber, I. Graz, S. Aazou, C. Ulbricht, D. A. M. Egbe, M. C. Miron, Z. Major, M. C. Scharber, T. Sekitani, T. Someya, S. Bauer, N. S. Sariciftci, Nat. Photonics 2013, 7, 811.

[22] J. Park, S. Wang, M. Li, C. Ahn, J. K. Hyun, D. S. Kim, D. K. Kim, J. A. Rogers, Y. Huang, S. Jeon, Nat. Commun. 2012, 3, 916.

[23] S. Chung, J. Lee, H. Song, S. Kim, J. Jeong, Y. Hong, Appl. Phys. Lett. 2011, 98, 153110.

[24] X. Hu, P. Krull, B. de Graff, K. Dowling, J. A. Rogers, W. J. Arora, Adv. Mater. 2011, 23, 2933.

[25] D.-H. Kim, J. Song, W. M. Choi, H.-S. Kim, R.-H. Kim, Z. Liu, Y. Y. Huang, K.-C. Hwang, Y. -w. Zhang, J. A. Rogers, Proc. Natl. Acad. Sci. 2008, 105, 18675. 
(a) Original size of display

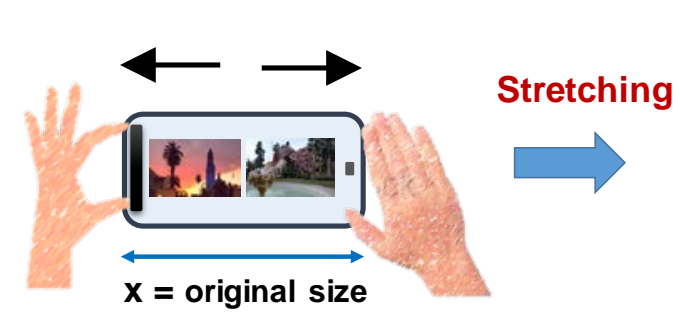

(c) Initial distribution of LEDs

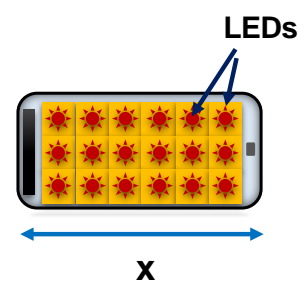

(b) Ideal resolution of display after stretching Fully expanded state

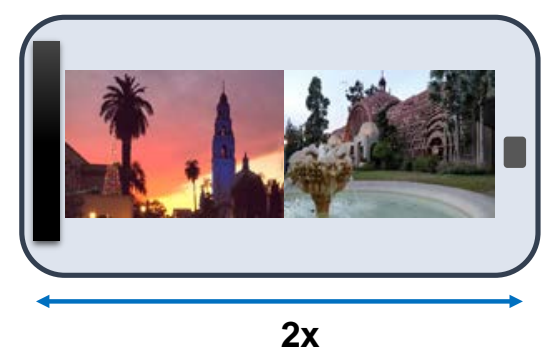

(d) Actual position of LEDs during stretching

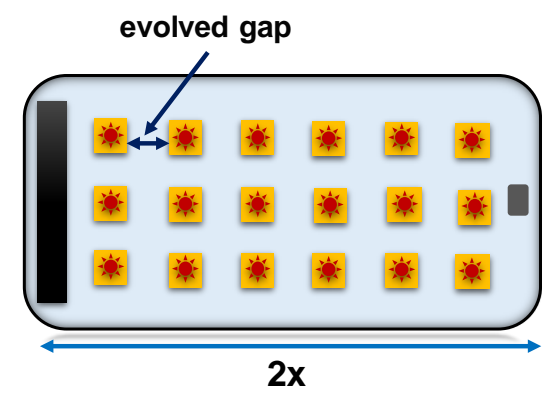

Figure 1. Expandable display and associated problem. (a, b) Conceptual outline of a representative reconfigurable display showing that the existing displays might expand to attain the enlarged screen or display, thus transform a mobile phone into Tab/IPAD or laptop. Effect of change in position of LEDs. (c) The quality or resolution of the image comprises of the LEDs position i.e. should have no gaps among the simultaneous LEDs, and (d) during stretching, the gap between LEDs starts to elevate and thus degrade the quality or resolution of the display. 
(a) Front view of reconfigurable platform

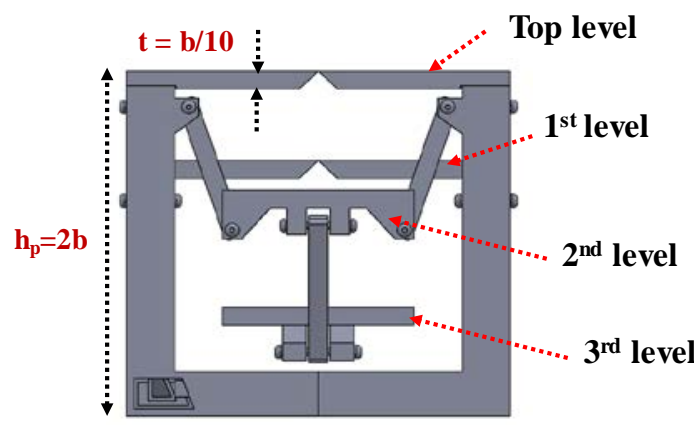

(c) 2 x 2 array

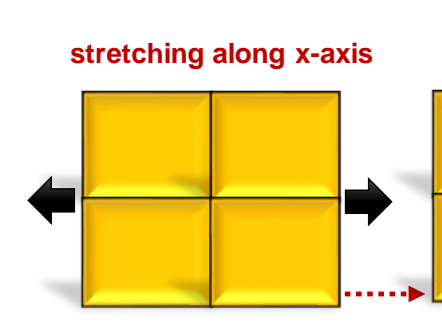

x 3 array (b) 3D outline of reconfigurable platform

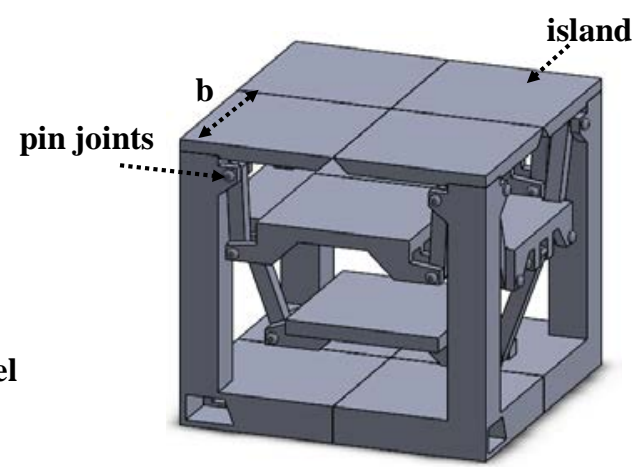

(e) $3 \times 3$ array (fully stretched) stretching along $y$-axis

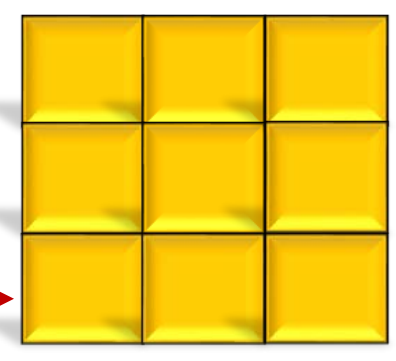

Figure 2. 3D design and underlying mechanism of our reconfigurable platform. (a) Front view and (b) demonstration of the fully assembled reconfigurable platform. The platform consists of multiple-levels, which open and move upward under the tensile forces along the $\mathrm{x}$ and $\mathrm{y}$-axis, respectively. The width and thickness of single square-island are represented as b and $t$, while total height of the platform is represented as hp, respectively. The working principle is demonstrated by the top view of the platform. (c) Initially, the platform consists of the 4 islands ( 2 x 2 array) called the top level and are under stretching along the $\mathrm{x}$-axis. (d) Stretching along the $\mathrm{x}$-axis results in the expansion of platform from $2 \times 2$ array into $2 \times 3$ array by moving the two islands of $1^{\text {st }}$ level outof-plane. (e) Stretching along the y-axis lifts up the remaining islands of $2^{\text {nd }}$ and $3^{\text {rd }}$ levels to acquire the $3 \times 3$ array (9 islands). The final configuration reveals that islands have no gaps among any consecutive islands. 


\section{WILEY-VCH}

(a) 3D-printed prototypes
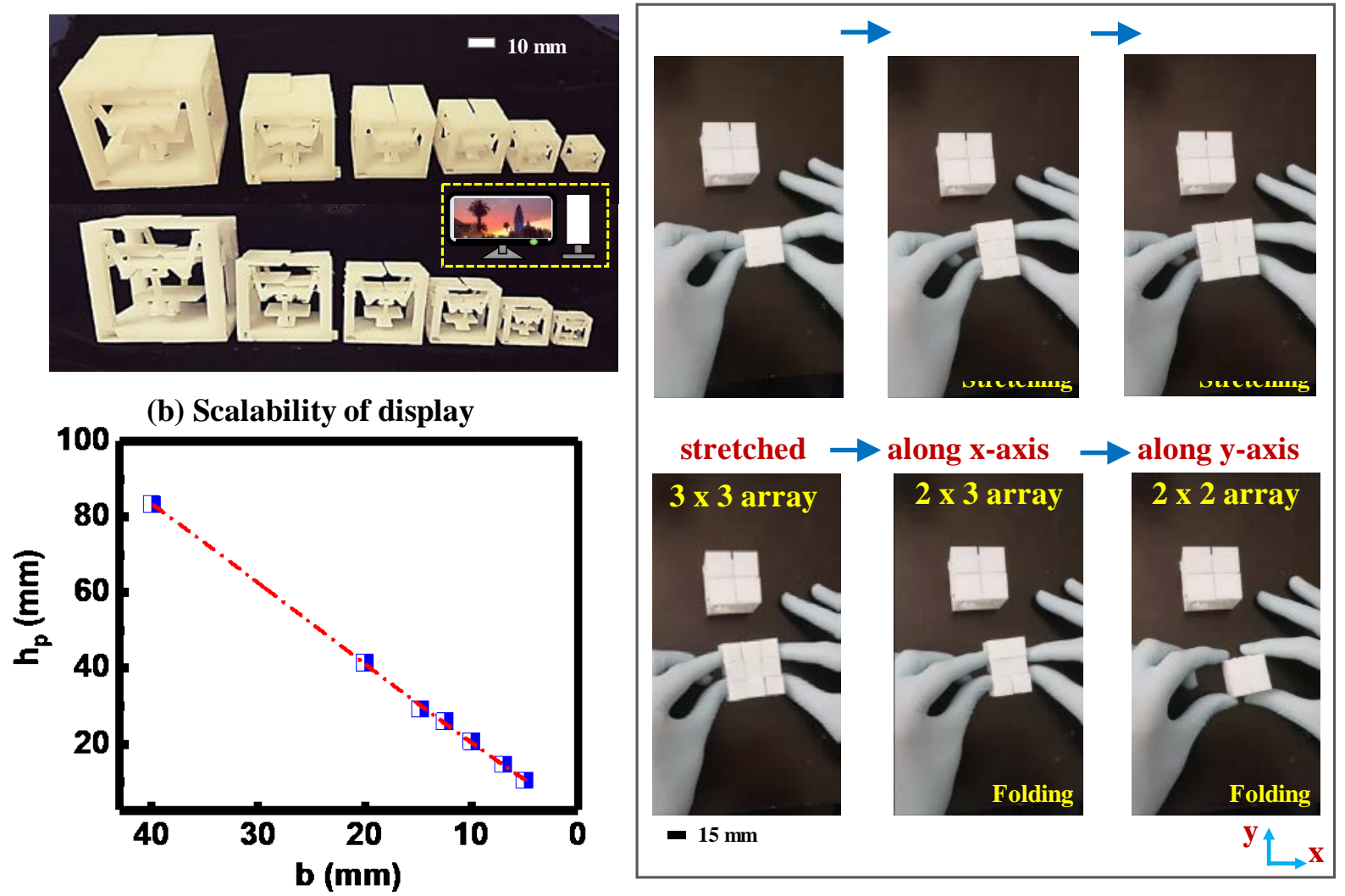

Figure 3. Proof-of-concept of the reconfigurable and expandable display by using the 3D printed platforms. (a) 3D printed and assembled platforms with different sizes ranging from $\mathrm{b}=$ $40 \mathrm{~mm}$ to $5 \mathrm{~mm}$. The smallest printed height of the platform is $10 \mathrm{~mm}$. For comparison, the front and cross section view of common display device is shown in inset. (b) A linear relationship between the height of platform $h_{p}$ and size of square island $b$ to exhibit the scalability of the design. (c) Reversible stretching and folding progression of the reconfigurable platform to demonstrate the transformation of the original 2x2 array (4 islands) into a 2x3 array (6 islands), and then into a $3 x 3$ array (9 islands), respectively and vice versa for folding. 


\section{WILEY-VCH}

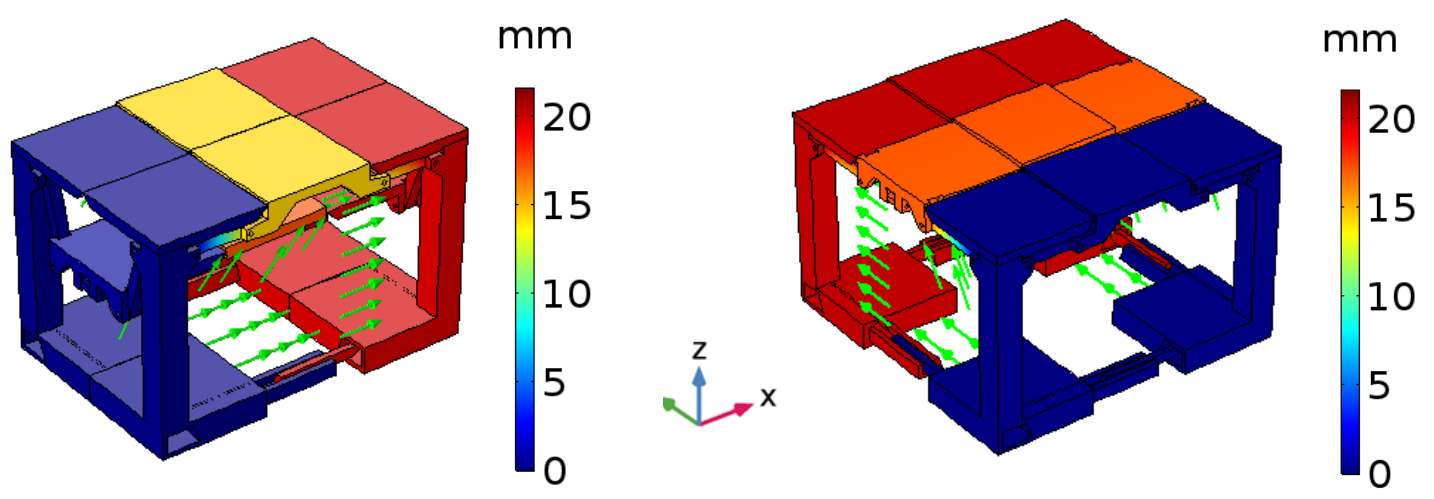

(c) Von Mises stress at fully stretched state

(a) Ivoual von ivisses stress nistory
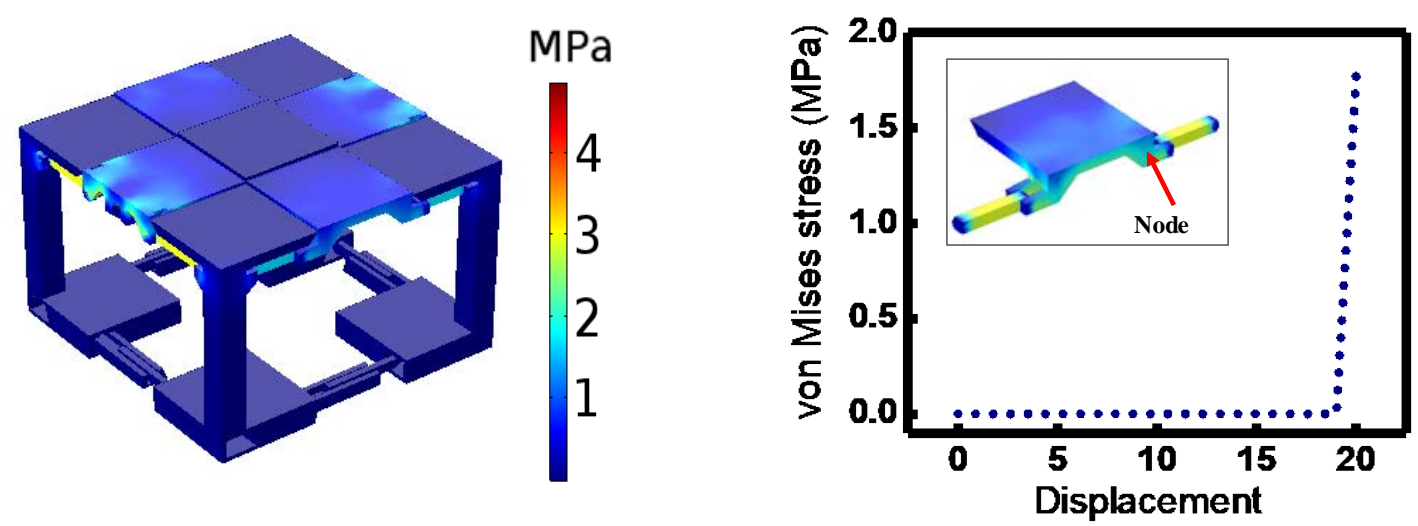

Figure 4. Numerical analysis of reconfigurable platform. (a) Displacement distributions under $\mathrm{x}$-axis stretching, which transform the 2x2 array into a 2x3 array, (b) stretching along the $y$-axis, which fully transforms into 3x3 array i.e. expresses the fully stretched state of the platform. The arrows show the path of movements of components. (c) The 3D von Mises stress distributions at the fully stretched state and (d) nodal stress history at one node of the link (marked in inset), show that the induced stresses are negligible since stress amount is in MPa range. Lower stresses ensure the mechanical reliability by avoiding the crack-based failure that usually occurs at higher stresses ( in the range of GPa). 


\section{WILEY-VCH}

(a) $2 \times 2$ array

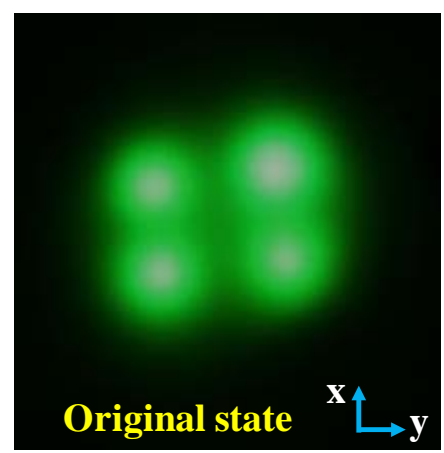

(d) $3 \times 3$ array

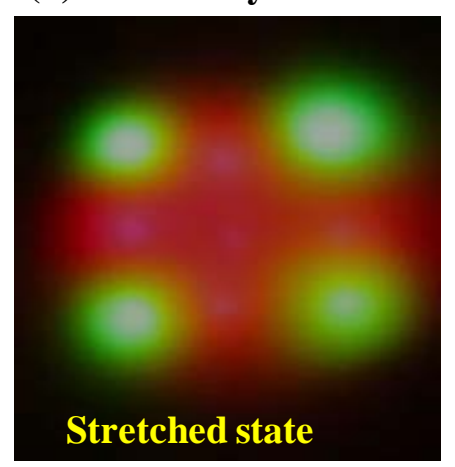

(b) 2 x 3 array

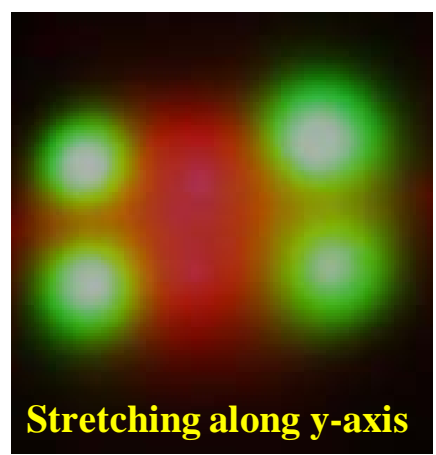

(e) $2 \times 3$ array

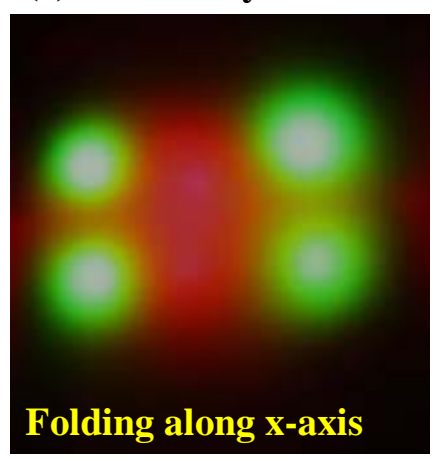

(c) $3 \times 3$ array

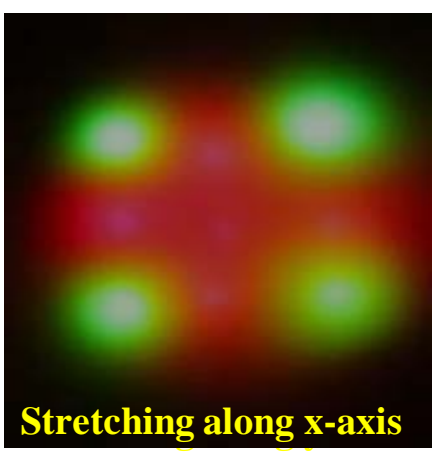

(f) 2 x 2 array

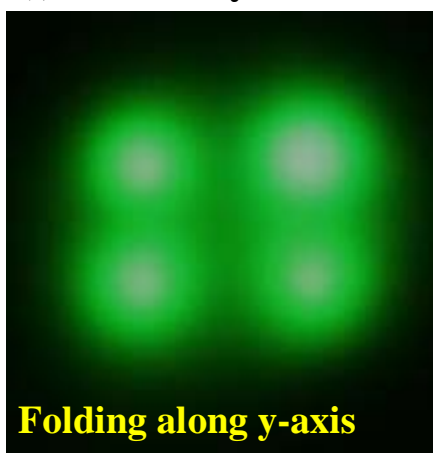

Figure. 5. Reversible and reconfigurable platform with LEDs. (a-c) During stretching, size of the display increases from the 2x2 array to 2x3 array and then to the $3 \times 3$ array, respectively. The resolution of the display is preserved by the LEDs moving upward or out-of-plane. These LEDs are placed onto the islands of $1^{\text {st }}-3^{\text {rd }}$ level. (d-f) Folding of platform reverts from the enlarged size of LEDs display from the 3x3 array to 2x3 and then 2x2 array, respectively. 


\section{WILEY-VCH}

\section{Table of Contents}

A reversible and reconfigurable display is proposed, which utilizes the novel concept of multilevel arrangement of LEDs. The proposed platform caters the problem of distorted display/image quality during stretching by preserving the actual resolution of the display. It also does not change the resistance of LEDs. 3D printed platform was tested and compared with the FEM results.
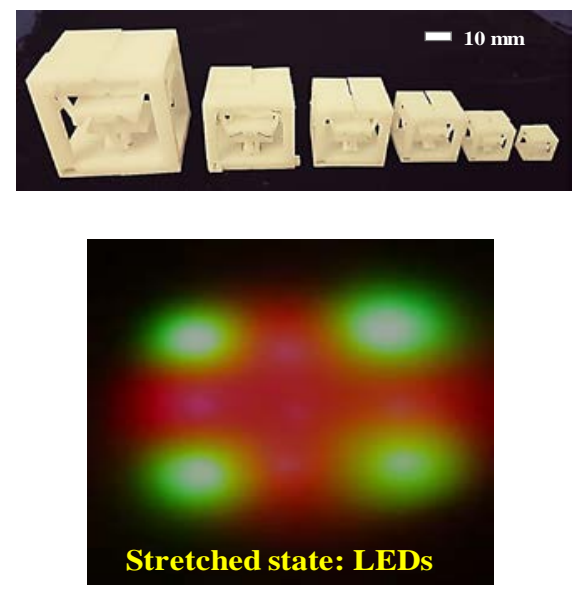

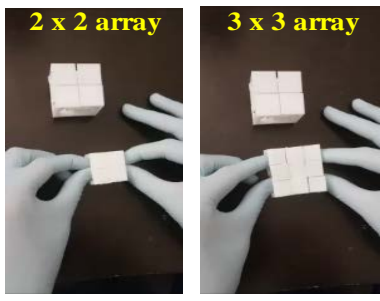

von Mises stress( MPa)

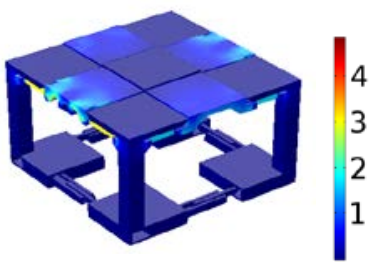




\section{Supplementary Information}

\section{D Printed Robotic Assembly Enabled Reconfigurable Display with Higher Resolution}

Nadeem Qaiser, Sherjeel Munsif Khan, Kelvin Chow, Marlon Diaz Cordero, Irmandy Wicaksono and Muhammad Mustafa Hussain ${ }^{1 *}$

${ }^{1}$ mmh labs, Electrical Engineering, Computer Electrical and Mathematical Science and Engineering Division, King Abdullah University of Science and Technology (KAUST)

Thuwal, 23955, Saudi Arabia, Tel.: +966-544-700-072

*Corresponding author; E-mail: muhammadmustafa.hussain@kaust.edu.sa

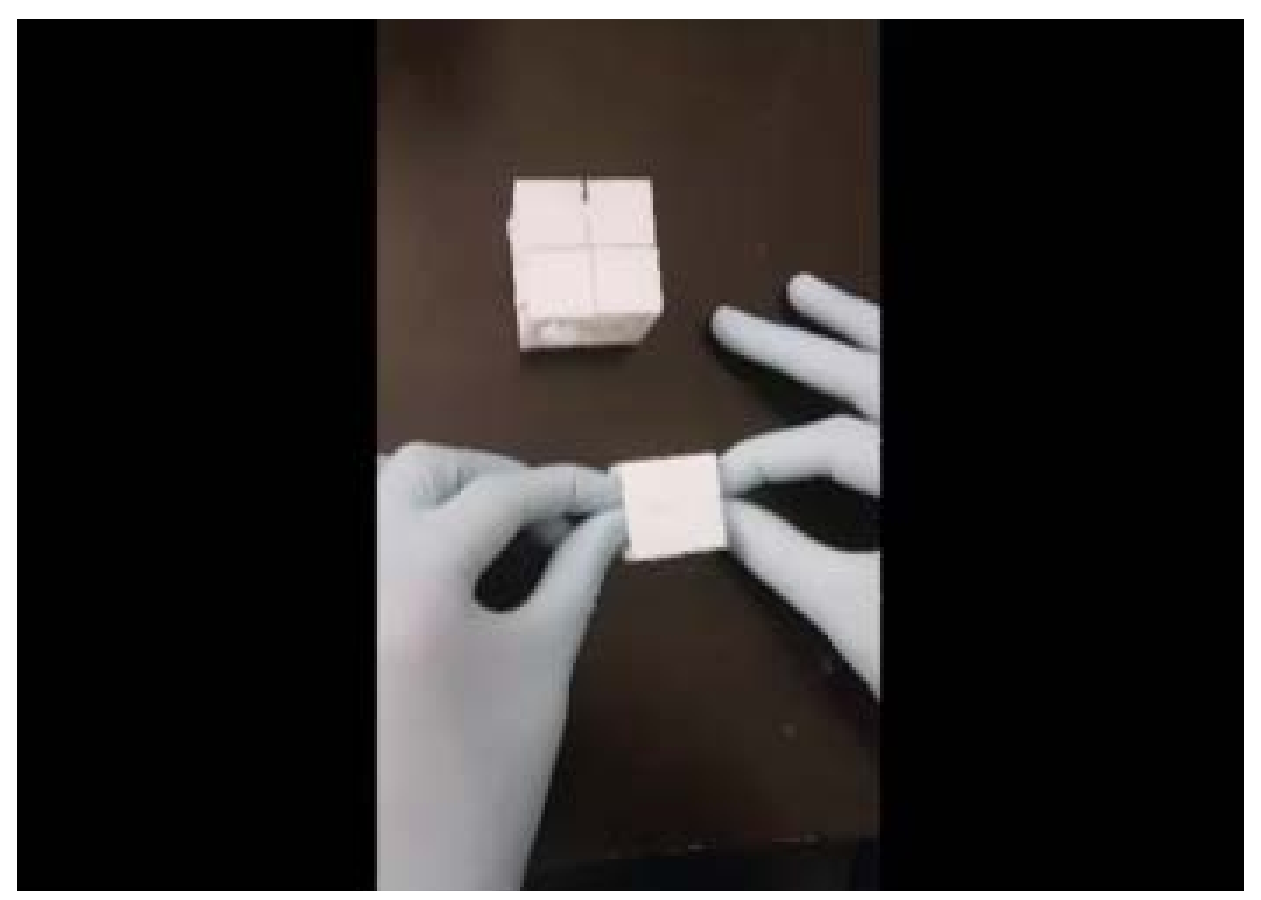

Figure SMovie1. Stretching and folding process of 3D printed reconfigurable platform 


\section{WILEY-VCH}

(a) Strain at fully stretched state
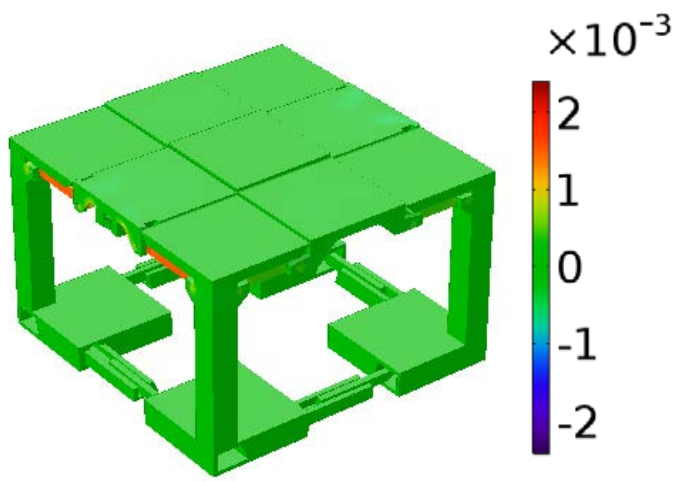

(b) 3D contours of von Mises stress at fully stretched state

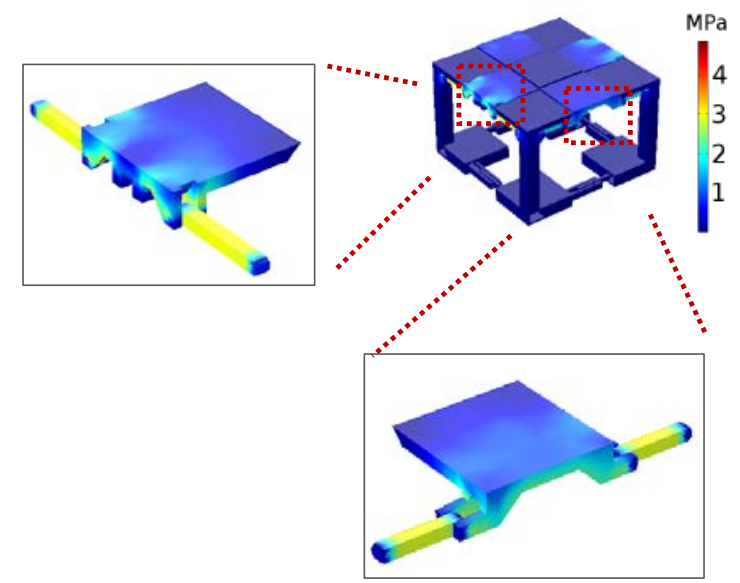

Figure S1. (a) 3D strain distributions of the platform and (b) detailed 3D stress contours of pins and links at the end of stretching process.

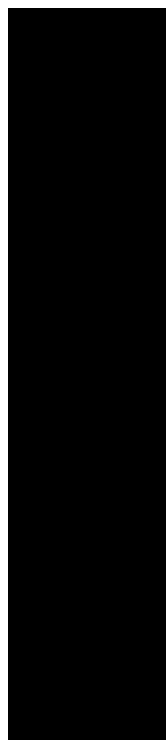

\section{From $2 \times 2$ to $2 \times 3$ array}

$\mathrm{mm}$
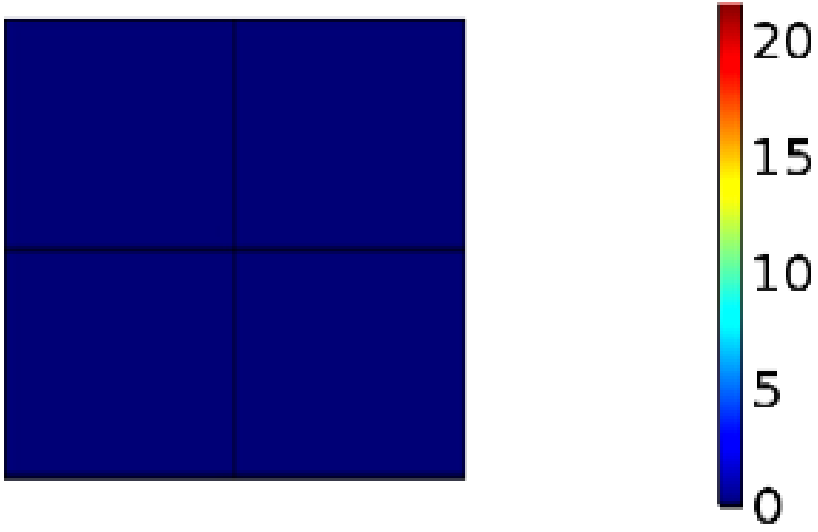

Figure SMovie2. Top view for the transformation of the 2x2 array into (a) 2x3 array, (b) $3 \times 3$ array by using the FEM. 


\section{WILEY-VCH}

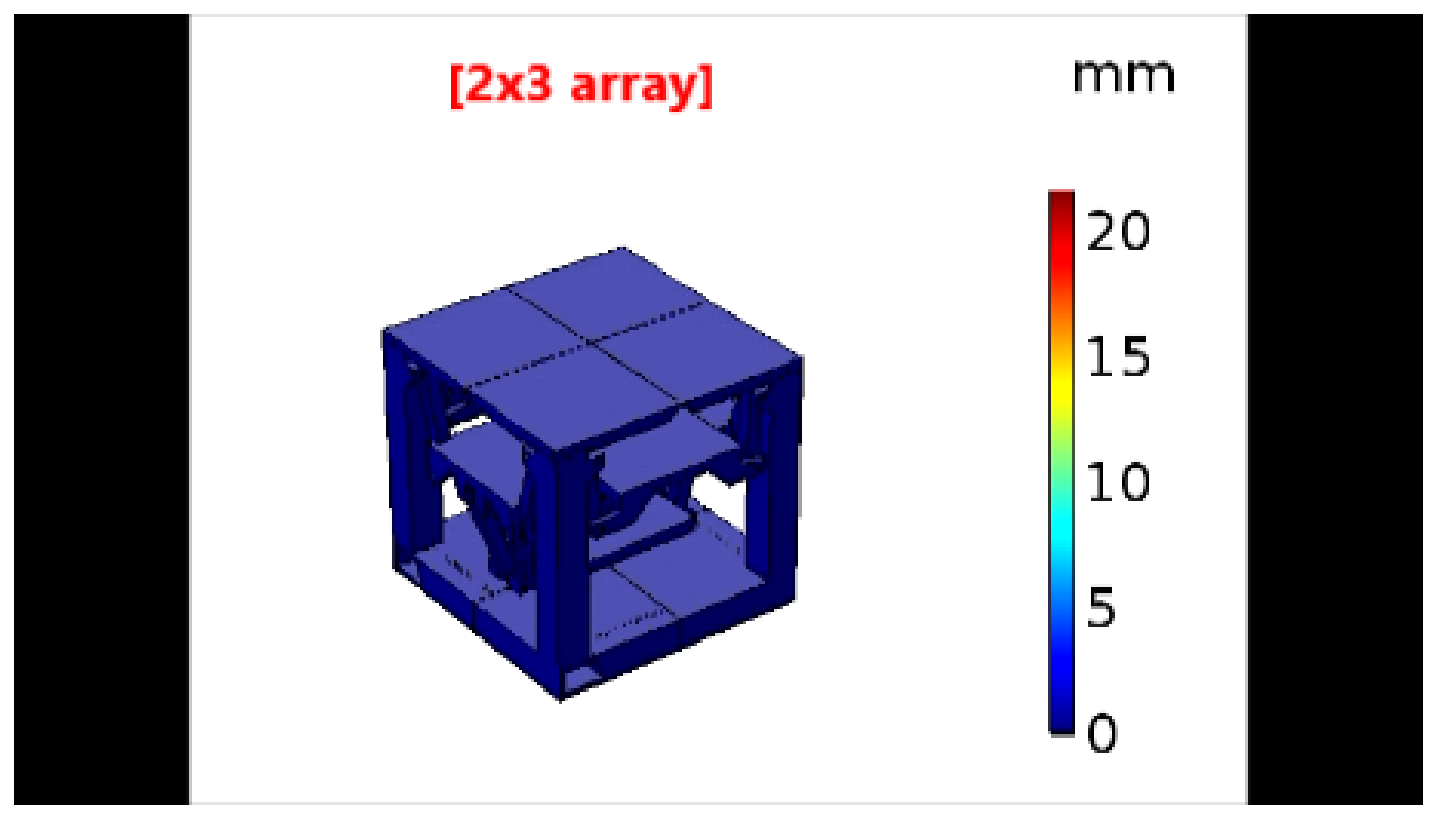

Figure SMovie3. Numerical results for stretching, folding process, and stress in link-island of the reconfigurable platform.

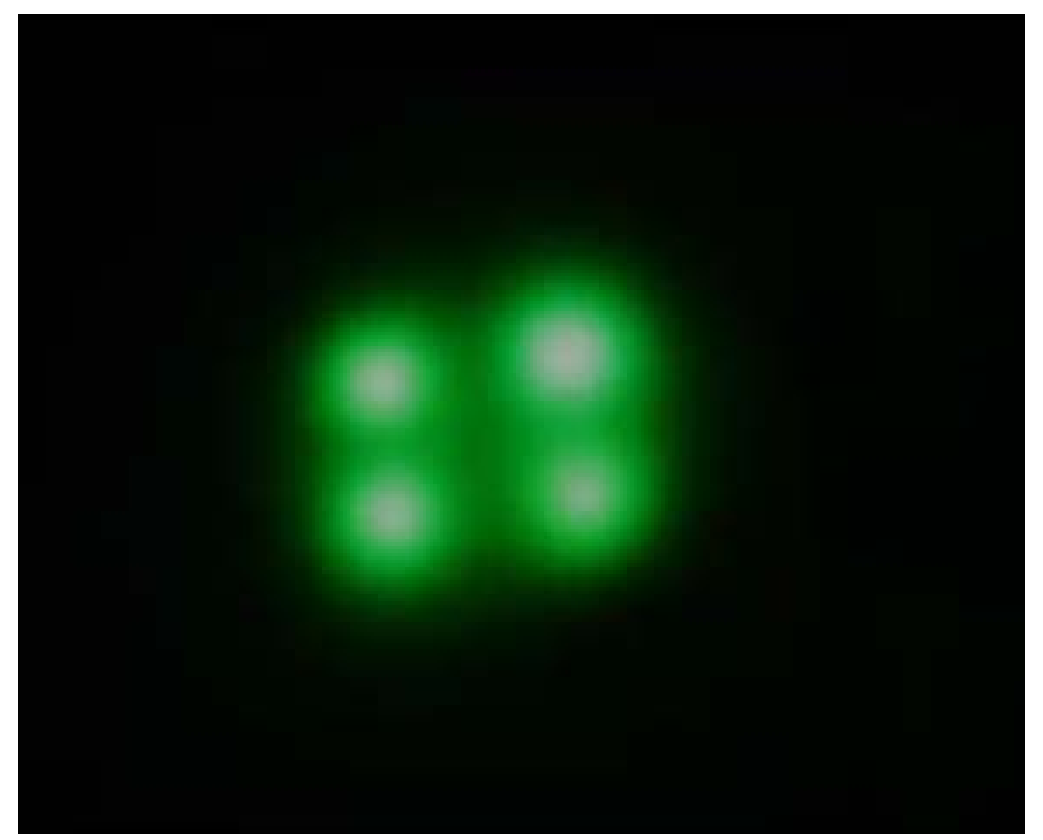

Figure SMovie4. Fully functional LEDs based 3D printed reconfigurable display. 